\section{Military Technical College}

Kobry El-Kobbah,

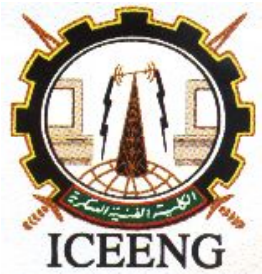

\author{
$10^{\text {th }}$ International Conference \\ on Electrical Engineering
}

ICEENG 2016

\title{
Nuclear Reactors Safety Core Parameters Prediction using Artificial Neural Networks
}

\author{
Amany S. Saber, Moustafa S. El-Koliel \\ Nuclear Research Center, \\ Atomic Energy Authority, \\ Cairo, Egypt \\ amanyss@hotmail.com
}

\author{
Mohamed A. El-Rashidy, Taha E. Taha \\ Faculty of Electronic Engineering, \\ Menoufiya University, \\ Cairo, Egypt
}

\begin{abstract}
The present work investigates an appropriate algorithm based on Multilayer Perceptron Neural Network (MPNN), Apriori association rules and Particle Swarm Optimization (PSO) models for predicting two significant core safety parameters; the multiplication factor $K_{\text {eff }}$ and the power peaking factor $P_{\max }$ of the benchmark 10 MW IAEA LEU research reactor. It provides a comprehensive analytic method for establishing an Artificial Neural Network (ANN) with selforganizing architecture by finding an optimal number of hidden layers and their neurons, a less number of effective features of data set and the most appropriate topology for internal connections. The performance of the proposed algorithm is evaluated using the 2-Dimensional neutronic diffusion code MUDICO-2D to obtain the data required for the training of the neural networks. Simulation results demonstrate the effectiveness and the notability of the proposed algorithm comparing with Trainlm-LM, quasiNewton (Trainbfg-BFGS), and Resilient Propagation (trainrpRPROP) algorithms.
\end{abstract}

Keywords: Apriori Association Rules; Particle Swarm Optimization; Artificial Neural Networks; Effective Multiplication Factor; and Power Peaking Factor.

\section{INTRODUCTION}

Emulating the calculations with Artificial Neural Networks (ANNs) in the field of nuclear engineering has been widely used in recent years. ANNs were applied successfully as an advanced tool for simulating several reactor physics parameters $[1,2,3,4,5,6]$. Fuel management scheme is a branch of nuclear engineering, which aims to determine the best fuel arrangements throughout a nuclear fuel cycle. The fuel-reloading process has important effect on both safety and economics. Finding the optimum configuration of fuel assemblies requires a huge amount of calculations in classical methods. Classical methods are not capable of performing this amount of calculations in a reasonable time. For complicated tasks like those associated with the nuclear fuel management optimization problem where an enormous number of possible arrangements exist in the available search space, the use of Artificial intelligence (AI) is a growing need. The main purpose in using ANN in a nuclear fuel management optimization is to reduce the computation time usually needed in such optimization processes and also to present comprehensive exploration to find endurable good configurations. The proposed algorithm is presented to find the optimal neural network architecture for predicting core safety parameters, such as multiplication factor $K_{\text {eff }}$ and power peaking factor $P_{\max }$, in nuclear research reactors. This was carried out through functioning design process that includes new strategy for learning algorithm to select effective features of the data sets, find an optimal number of hidden layers and their neurons, and topology connections. In [2] and [7], ANN architecture is used in predicting core safety parameters. However, even though the predictive results achieved were globally satisfactory, the convergence during the training process was time-consuming for investigated parameters.

The configuration of the neural network depends highly on the problem. Thus, a suitable architecture is determined for 
each application by the trial and error. It is often necessary to perform a lot of experiments that are time-consuming to get the optimal architecture and parameter settings for the neural networks, and the performance of the neural network relates highly to the experience of the users. The choice of the number of hidden nodes and the weights initialization are a crucial problem for a multilayer neural network. In general, the number of hidden units is associated with the complexity of the functional mapping from the input to the output.

In general, too large a network may tend to overfit the training data and affect the generalization capability, whereas too small a network may not even be able to learn the training samples due to its limited representation capability. In addition, a fixed structure of overall connectivity between neurons may not provide the optimal performance within a given training period. Therefore, some attention has been given to the problem of how to construct a suitable network structure for a given task. Another remaining issue in designing the networks is to determine the initial weights that make the neural network convergence fast and improve neural network generalization capabilities. Many researchers have paid attention to the optimization of network structure and connection weights. References [8] and [9] proposed a modification approach to initialize the Neural Network (NN) weights and to determine the optimal number of hidden nodes for $\mathrm{NN}$. Reference [10] presented an improved genetic algorithm to tune the structure and parameters simultaneously. In [11], a hybrid Taguchi-genetic algorithm is used to solve the problem of tuning both the network structure and parameters. In [12], a novel Hybrid Higher Order Neural Classifier (HHONC) based on HONN models is introduced, and then using the proposed classifier over various benchmark statistical datasets. A hybrid model Partial Least Square Neural Network (PLSNN) which combines PLS and $\mathrm{NN}$ is developed in [13] to enhance the detection performance, and also a Quantum-based Neural Network (QNN) is proposed in [14].

The proposed algorithm can reach to a consolidated structure size, which it based on a priori knowledge during several training phases, assisted by Apriori algorithm of association rules, and it used particle swarm algorithm for an optimization process to minimize the number of training phases. This algorithm is considered as initiative step for using this work as computational tool for an optimal fuel management in nuclear reactors. It is developed on the benchmark IAEA (International Atomic Energy Agency) 10 MW benchmark LEU (Low Enriched Uranium) core (IAEA TECDOC-643, 1992) [15].

The developed 2-Dimensional neutronic diffusion code MUDICO-2D [16] is used to obtain the data required for the training of the neural networks. The structure of this paper is as follows. In section 2, the reactor core model is introduced. In section 3, a description of the proposed algorithm will be detailed. In section 4, the extensive experimental results of proposed algorithm will be discussed. In the last section, the concluding remarks will be offered.

\section{IAEA $10 \mathrm{MW}$ BENCHMARK REACTOR CORE}

Due to restricted trade in High Enrichment Uranium (HEU) based fuel; the reactors around the world are being converted to use LEU based fuels. In order to provide

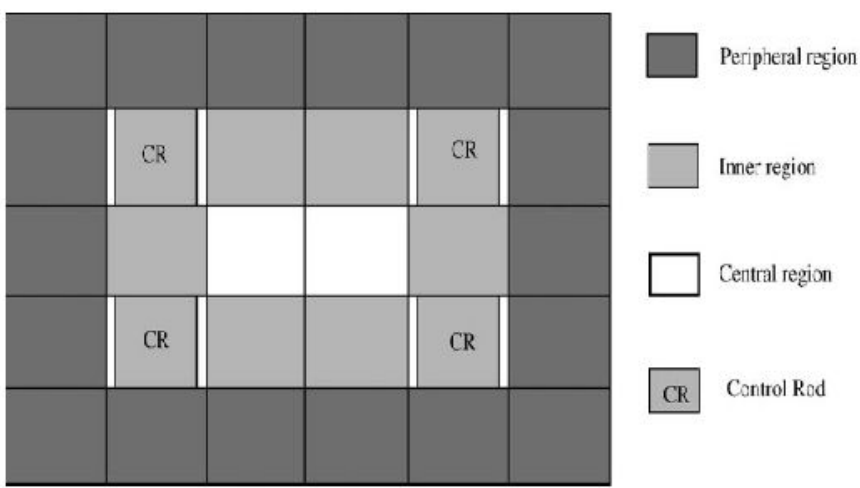

guidelines and facilitate the reactor conversion from HEU to LEU fuel, IAEA devised a standard benchmark Material Test Reactor (MTR). The reactor is a 10 MW tank-in-pool, Material Test Reactor (MTR). It has been devised to facilitate the conversion of MTR's fuelled with HEU based fuels to use LEU based fuels.

The reactor core is an arrangement of $5 \times 6$ matrix having 21 standard fuel elements of 23 plates each and 4 control fuel elements with 17 plates. It is reflected by graphite on two opposite sides parallel to fuel plates, with thickness of one fuel element. The core is immersed in light water. There are five flux traps, one at the center of the core and one each at the four corners of the core as shown in Fig.1 [17]. For calculation needs, a small modification was introduced in its initial core arrangement, to guarantee geometrical asymmetry in the core and subsequently to obtain the sufficient search space.

A total of 30 positions were considered. For better representation of various states of the reactor core during its useful life, ranging from its beginning of life to the end of life, several configurations have been taken into account and emulated using both standard and control fuel element with different burn-up, ranging from fresh fuel (FF) to $50 \%$ (FF, $5 \%, 10 \%, 25 \%, 30 \%, 45 \%, 50 \%)$ for the first one and from fresh fuel to $30 \%$ (FF, $25 \%, 30 \%$ ) for the second one. The reactor core was then transformed into a vector form of 30 positions so that it can be presented to the networks as input values. 
Fig. 1: $10 \mathrm{MW}$ IAEA benchmark research reactor core

The reactor core was modeled using the 2-Dimensional neutronic diffusion code MUDICO-2D for the referenced target values, and using several established inference rules to prevent both high power regions and high reactivity difference. Finally, a total of 572 core configurations have been created, in which 542 were used for the training process, whereas the remaining set was used to test the developed network performance. The inference rules stated are introduced in terms of restrictions and constraints that will not be contravened, during the optimization process; otherwise the safety margin of the reactor should be provoked. These heuristics are described as follow:

- Fresh fuel elements cannot be moved into central positions of the core as shown in Fig.2 [3].

- An exchange between fresh fuel and highest burned fuel elements (45 and 50\%) is prohibited.

- Highest burned fuel elements should not be moved into the peripheral region.

- Control fuel elements are in fixed positions.

- Only one type of control elements should be present in the core during one cycle.

- Foursomes assembled fresh fuel elements are excluded.

Fig. 2: Reference core arrangement

\section{PROPOSED ALGORITHM}

We have two externally supplied inputs; the first input is the given training data set which is denoted by $\mathrm{T}$, and the second input is empty data set denoted by $\mathrm{D}$. The inputs to proposed algorithm can help to formalize the requirements and restrictions that the architecture must fit. The quantities produced after applying the proposed algorithm are the outputs which are organized as follows: the number of hidden layers of neural network architecture is denoted by $\mathrm{J}$, the number of nodes for each hidden layer $\mathrm{J}$ is denoted by $\mathrm{I}$, the term TOP is used to represent neural network topology, the term RMSE is used to measure the root mean square error of the network, and finally the overall classification accuracy of the network is denoted by ACC. The goal of this algorithm is to establish an optimal artificial neural network structure with self-organizing architecture included the most effective input features, number of hidden layers and their hidden neurons, network topology. It can reach a compact structure size, based on a priori knowledge from a set of training samples, assisted by Apriori algorithm for association rules and particle swarm for optimization process. The proposed algorithm is organized in 16 steps as shown in Fig. 3.

Input: Training data set $\mathrm{T}$, and empty data set $\mathrm{D}$.

Output: J, I, TOP, RMSE, and ACC number of hidden layers, number of nodes for hidden layer J, neural network topology, root mean squared error, and overall accuracy, respectively.

Step 1: Initialize original data: by normalization process to form training data set.

Step 2: Initialize neural network architecture: included $\mathrm{H}_{\mathrm{J}}$, and $\mathrm{N}_{\mathrm{Ji}}$ to set $\mathrm{J}=0$, and $\mathrm{i}=0$.

Step 3: Update D: to equal T.

Step 4: Set iterative variable: $\mathrm{J}=\mathrm{J}+1$.

\begin{tabular}{|c|c|c|c|c|c|c|}
\hline W & G & \multicolumn{2}{|l|}{ G } & G & G & w \\
\hline W & $5 \%$ & \multicolumn{2}{|c|}{$25 \%$} & $\begin{array}{l}\text { SFE-2 } \\
25 \%\end{array}$ & $\begin{array}{c}\text { SFE-1 } \\
\mathbf{5} \%\end{array}$ & w \\
\hline $5 \%$ & $25 \%$ & \multicolumn{2}{|c|}{$45 \%$} & $\begin{array}{l}\text { SFE-3 } \\
45 \%\end{array}$ & $\begin{array}{c}\text { CFE-1 } \\
25 \%\end{array}$ & $5 \%$ \\
\hline $25 \%$ & $45 \%$ & $45 \%$ & H2O & $45 \%$ & $45 \%$ & $25 \%$ \\
\hline $5 \%$ & $25 \%$ & $45 \%$ & & $45 \%$ & $25 \%$ & $5 \%$ \\
\hline $\mathbf{w}$ & $5 \%$ & $25 \%$ & & $25 \%$ & $5 \%$ & W \\
\hline W & G & G & & G & G & W \\
\hline
\end{tabular}

Fig. 3: Pseudo code of proposed algorithm.

First normalization process is applied to reduce redundancies of data in database, and manipulate attributes in a desired appropriate form. An initial $\mathrm{NN}$ is then created consisting of: input layer, $\mathrm{H}_{\mathrm{J}}\left(\mathrm{N}_{\mathrm{Ji}}\right.$ neurons), and output layer, network topology is not recognized yet. Specifying minimum support and minimum confidence; Apriori algorithm finds the most effective sets of related data set inputs (features). Each extracted features set $S_{i}$ construct a hidden node $\mathrm{N}_{\mathrm{Ji}}$, where features of this set $\mathrm{S}_{\mathrm{i}}$ are input of the new constructed hidden node $\mathrm{N}_{\mathrm{Ji}}$. So, all possible hidden nodes are equal to discovered sets $S_{i}$ and their features are input for constructed nodes to build network topology, which leads to minimizing in the connections.

PSO algorithm is used to find an optimum number of hidden nodes in each hidden layer which achieves highest accuracy, thus by determining the best values of support and confidence that give the highest performance for the structured network. A dynamic strategy is used to train neural network; this strategy can insert or remove hidden nodes and layers, and it can change topology of neural network via the measurement of classification accuracy in the training process. Classification accuracy and root mean square error (RMSE) are used to decide whether neurons should be inserted or deleted. Initially, a network with one hidden layer and one hidden neuron is constructed, and then 
new hidden neurons (only one neuron is inserted at a time) are incrementally changed. Each new hidden neuron and hidden layer is trained to minimize the current network error in order to enhance the performance of the network. A pruning approach is applied to enhance the selection, and remove the unnecessary hidden neurons (or connections) either during training or after convergence to a local minimum. Finally, we stop when pre-specified error requirement is found, or no performance refinement is noticed.

\section{EXPERIMENTAL RESULTS}

Experimental studies are presented to prove the effectiveness and the notability of proposed algorithm. We applied our proposed method to predict two safety parameters $\left(K_{\text {eff }}\right.$ and $\left.P_{\max }\right)$ of a benchmark 10 MW IAEA LEU core research reactor, where they are important for attempting to optimize the performance of the reactor. For each parameter, we normalized the data over each feature value. First step, construct an optimized network by applying our proposed algorithm using the desired database. The information required in the related database will contain a coupled input value with the corresponding target output values. These values are used to train the networks until the error reaches a desired value. Several experiments have been executed by varying the two parameters confidence $(C O N F$.) and support (SUPP.) that measure the strength of association rules. PSO determines the optimal values of CONF. and SUPP. that achieve the highest performance for the structured network.

Once a network has been structured it is ready to be trained. Hence, a supervised training has been carried out for each parameter with both the inputs and their associated outputs provided. The constructed network processes the inputs and compares its resulting outputs against the desired outputs. A total of 542 configurations were used for the training process. The training results for the multiplication factor $K_{\text {eff }}$, and power peaking factor $P_{\max }$ are presented in Tables I and II respectively. After training (Second step), the constructed network is created for each parameter and tested against the unseen data. 30 configurations were devoted to this purpose. The prediction results for $K_{\text {eff }}$ and $P_{\max }$ parameters were shown in Tables III, IV respectively. The altitude of classified accuracy using proposed model can be appeared by sensitive rates, which compared with Levenberg - Marquardt (Trainlm-LM), quasi-Newton (Trainbfg-BFGS), and Resilient Propagation (trainrp $R P R O P$ ) algorithms [17]. The reported results in tables $\mathrm{V}$, VI are averaged over 50 trials of experiments. The averaged values of the following parameters were used to measure the accuracy, number of selected features, number of preserved nodes in the hidden layers and mean accumulated CPU time the training process taken.

\begin{tabular}{|c|c|c|c|c|c|c|c|}
\hline CONF. & SUPP. & $\begin{array}{c}\text { No. of Selected } \\
\text { Features }\end{array}$ & $\begin{array}{c}\text { No. of Hidden } \\
\text { Layers }\end{array}$ & $\begin{array}{c}\text { No. of Hidden } \\
\text { Nodes }\end{array}$ & RMSE & $\begin{array}{c}\text { Performance } \\
\text { \% }\end{array}$ & $\begin{array}{c}\text { Time } \\
\text { (S) }\end{array}$ \\
\hline $\mathbf{0 . 9}$ & $\mathbf{0 . 1 5}$ & $\mathbf{7}$ & $\mathbf{1}$ & $\mathbf{1 0}$ & $\mathbf{0 . 0 2 2 8}$ & $\mathbf{7 0 . 6 6}$ & $\mathbf{0 . 3 3}$ \\
\hline 0.7 & 0.25 & 3 & 1 & 2 & 0.0263 & 60.7 & 0.27 \\
\hline 0.6 & 0.35 & 6 & 1 & 14 & 0.0234 & 58.86 & 0.33 \\
\hline 0.5 & 0.55 & 2 & 1 & 3 & 0.0271 & 59.23 & 0.33 \\
\hline 0.3 & 0.4 & 3 & 1 & 4 & 0.0269 & 59.23 & 0.31 \\
\hline
\end{tabular}

TABLE I.

Training results for the multiplication factor $K_{e f f}$ 


\begin{tabular}{|c|c|c|c|c|c|c|c|}
\hline CONF. & SUPP. & $\begin{array}{c}\text { No. of Selected } \\
\text { Features }\end{array}$ & $\begin{array}{c}\text { No. of Hidden } \\
\text { Layers }\end{array}$ & $\begin{array}{c}\text { No. of Hidden } \\
\text { Nodes }\end{array}$ & RMSE & $\begin{array}{c}\text { Performance } \\
\text { \% }\end{array}$ & $\begin{array}{c}\text { Time } \\
\text { (S) }\end{array}$ \\
\hline $\mathbf{0 . 9}$ & $\mathbf{0 . 0 5}$ & $\mathbf{9}$ & $\mathbf{1}$ & $\mathbf{2 5}$ & $\mathbf{0 . 0 9 1 7}$ & $\mathbf{8 8 . 1 9 2}$ & $\mathbf{0 . 2 2}$ \\
\hline 0.5 & 0.1 & 5 & 1 & 6 & 0.1367 & 75.28 & 0.2 \\
\hline 0.3 & 0.15 & 5 & 1 & 12 & 0.1375 & 75.28 & 0.19 \\
\hline 0.2 & 0.2 & 2 & 1 & 2 & 0.1404 & 70.11 & 0.2 \\
\hline
\end{tabular}

TABLE III. Testing results for the multiplication factor $K_{e f f}$

\begin{tabular}{|c|c|c|c|c|c|c|c|}
\hline CONF. & SUPP. & $\begin{array}{c}\text { No. of Selected } \\
\text { Features }\end{array}$ & $\begin{array}{c}\text { No. of Hidden } \\
\text { Layers }\end{array}$ & $\begin{array}{c}\text { No. of Hidden } \\
\text { Nodes }\end{array}$ & $\begin{array}{c}\text { RMSE } \\
\text { Performance } \\
\text { \% }\end{array}$ & $\begin{array}{c}\text { Time } \\
\text { (S) }\end{array}$ \\
\hline $\mathbf{0 . 9}$ & $\mathbf{0 . 1 5}$ & $\mathbf{7}$ & $\mathbf{1}$ & $\mathbf{1 0}$ & $\mathbf{0 . 0 2 4 5}$ & $\mathbf{8 3 . 3 3}$ \\
\hline 0.7 & 0.25 & 3 & 1 & 2 & 0.0234 & 80.07 \\
\hline 0.6 & 0.35 & 6 & 1 & 14 & 0.0254 & 70 \\
\hline 0.5 & 0.55 & 2 & 1 & 3 & 0.0244 & 0.02 \\
\hline 0.3 & 0.4 & 3 & 1 & 4 & 0.0244 & 73.33 \\
\hline
\end{tabular}

TABLE IV. Testing results for the power peaking factor $P_{\max }$

\begin{tabular}{|c|c|c|c|c|c|c|c|}
\hline CONF. & SUPP. & $\begin{array}{c}\text { No. of Selected } \\
\text { Features }\end{array}$ & $\begin{array}{c}\text { No. of Hidden } \\
\text { Layers }\end{array}$ & $\begin{array}{c}\text { No. of Hidden } \\
\text { Nodes }\end{array}$ & $\begin{array}{c}\text { RMSE } \\
\text { Performance } \\
\text { \% }\end{array}$ & $\begin{array}{c}\text { Time } \\
\text { (S) }\end{array}$ \\
\hline $\mathbf{0 . 9}$ & $\mathbf{0 . 0 5}$ & $\mathbf{9}$ & $\mathbf{1}$ & $\mathbf{2 5}$ & $\mathbf{0 . 1 1 7 1}$ & $\mathbf{9 3 . 3 3}$ \\
\hline 0.5 & 0.1 & 5 & 1 & 6.02 & 0.1795 & 86.67 \\
\hline 0.3 & 0.15 & 5 & 1 & 12 & 0.1806 & 86.67 \\
\hline 0.2 & 0.2 & 2 & 1 & 2 & 0.03 & 83.33 \\
\hline
\end{tabular}

TABLE V. Comparison of selected features, hidden nodes number, and performance

\begin{tabular}{|c|c|c|c|c|}
\hline & $\begin{array}{c}\text { No. of Selected } \\
\text { Features }\end{array}$ & $\begin{array}{c}\text { No. of Hidden } \\
\text { Nodes }\end{array}$ & Performance \% & Time (S) \\
\hline Proposed Algorithm $\boldsymbol{K}_{\text {eff }}$ & $\mathbf{7}$ & $\mathbf{1 0}$ & $\mathbf{8 3}$ & $\mathbf{0 . 0 3}$ \\
\hline LM & 30 & 15 & 83 & 4.98 \\
\hline BFGS & 30 & 20 & 62 & 112.92 \\
\hline RPROP & 30 & 25 & 38 & 31.53 \\
\hline
\end{tabular}


TABLE VI. Comparison of selected features, hidden nodes number, and performance

\begin{tabular}{|c|c|c|c|c|}
\hline & $\begin{array}{c}\text { No. of Selected } \\
\text { Features }\end{array}$ & $\begin{array}{c}\text { No. of Hidden } \\
\text { Nodes }\end{array}$ & Performance \% & Time (S) \\
\hline Proposed algorithm $\boldsymbol{P}_{\max }$ & $\mathbf{9}$ & $\mathbf{2 5}$ & $\mathbf{9 3}$ & $\mathbf{0 . 0 2}$ \\
\hline LM & 30 & 40 & 59 & 112.92 \\
\hline BFGS & 30 & 20 & 42 & 70.38 \\
\hline RPROP & 30 & 20 & 31 & \\
\hline
\end{tabular}

From the results in tables V and VI, it is clearly seen that, the proposed algorithm achieves the highest classification accuracy with lowest number of nodes in hidden layers which leads to provide the efficiency of treatment service with as little as possible in the decision making time after the construction of neural network topology. Besides, it is able to extract the most effective features of data sets. Minimizing in the connections and feature extraction are the main reasons of the improvement of training time of our algorithm comparing with the other self-organizing neural network and classifier algorithms which make the proposed algorithm faster than other approaches after reaching network structure by training phase.

Finally, after finishing our experiments, two MPNNs have been developed as an optimized structure, based on typical three layers (input - hidden - output) model. The first network predicts $K_{\text {eff }}$, and the second network predicts $P_{\max }$. The reactor core's 30 positions was transformed into a vector form which presented to the neural networks as input values to be treated, the input values have the following symbols (A1 to $A 30)$.

Fig. 4 shows the typical architecture of neural network that predicts $K_{\text {eff }}$, it contains 7 neurons as input (A2, A11, A12, A18, A20, A21, A28), 10 hidden neurons, and one neuron as output $\left(\right.$ Class_ $\left.\mathrm{K}_{\mathrm{eff}}\right)$. Fig. 5 shows the typical architecture of neural network that predicts $P_{\max }$, it contains 9 neurons as input (A1, A2, A10, A11, A12, A15, A18, A20, A30), 25 hidden neurons, and one neuron as output (Class_ $\left.P_{\max }\right)$.

As shown in Figs. 4, 5 not all 30 features are included, only 7, 9 features are extracted respectively, and minimizing in the connections are also shown.

\section{CONCLUSION AND FUTURE WORK}

In this paper, we have proposed a comprehensive algorithm based on Multilayer Perceptron Neural Network (MPNN) for predicting significant core safety parameters. The proposed algorithm can reach a consolidated structure size of artificial neural network with extracting most effective features, based on a priori knowledge from a set of training samples, assisted by Apriori algorithm for association rules and particle swarm for optimization process. Predicting two safety parameters $\left(K_{\text {eff }}\right.$ and $\left.P_{\max }\right)$ of a benchmark $10 \mathrm{MW}$ IAEA LEU core research reactor is used in order to show the suitability and effectiveness of the proposed model for classification tasks.

Simulation results show that the proposed algorithm achieves the highest classification accuracy with lowest number of nodes in hidden layers in smallest training time in comparison to all alternative classifier algorithms, besides, it is able to extract the most effective features. Further studies is using the ANN that predicts the $K_{\text {eff }}$ and the $P_{\max }$ as preceding step in the Simulated Annealing (SA) method to automate the distribution process for the fuel assemblies inside the reactor core.

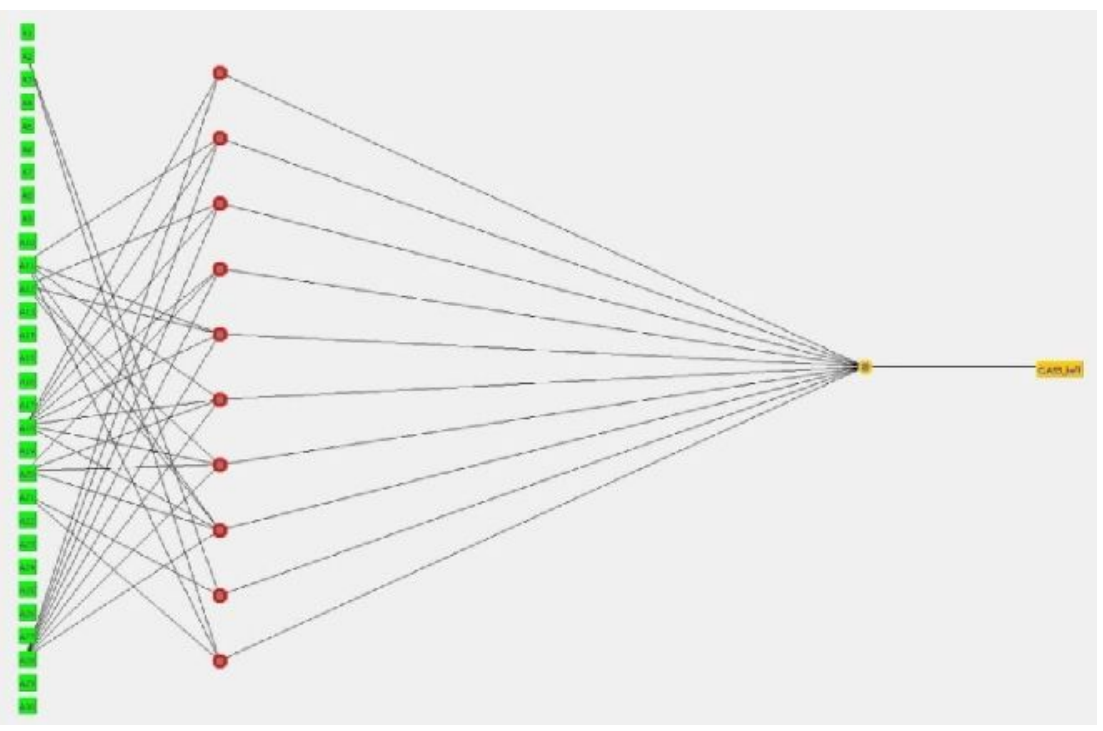


Fig. 4: Typical architecture of MPNN predicts $K_{\text {eff }}$

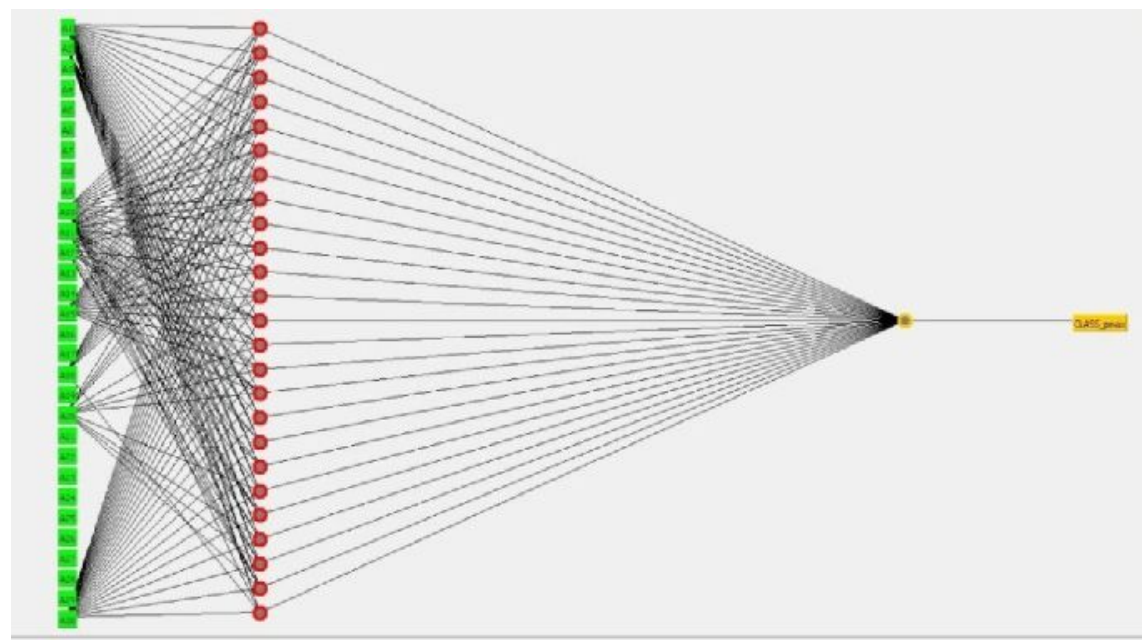

Fig. 5: Typical architecture of MPNN predicts $P_{\max }$

\section{REFERENCES}

[1] A.Erdogan, and M.Geckinli, "A PWR reload optimisation code (XCore) using artificial neural networks and genetic algorithms," Ann. Nucl. Energy, vol. 30, pp. 35-53, 2003.

[2] G. Kim, H. Chang, and H. Lee, "Pressurized water reactor core parameter prediction using an artificial neural network,"Nucl. Sci. Eng., vol. 113, pp. 70-76, 1993.

[3] H. Mazrou, and M. Hamadouche, "Development of a supporting tool for optimal fuel management in research reactors using artificial neural networks,” Nucl. Eng. Des.,vol. 236, pp. 255-266, 2006.

[4] F. Miller, A.Algutifan, and E.Uhrig, "An Application of Neural Networks and Artificial Intelligence for In-Core Fuel Management," Transactions of American Nuclear Society and the European Nuclear Society, Chicago, vol. 66, pp. 108-109, 1992.

[5] M.Sadighi, S.Setayeshi, and A.Salehi, "PWR Fuel Management Optimization Using Neural Networks,” Ann. Nucl. Energy, vol. 29, pp. 41-51, 2002.

[6] P. Souza, and L. Moreira, "Neural network correlation for power peak factor estimation,” Ann. Nucl. Energy, vol. 33, pp. 594-608, 2006.

[7] H. Mazrou, and M. Hamadouche, "Application of artificial neural network for safety core parameters prediction in LWRRS," Prog. Nucl.Energy, 44 (3), pp. 263-275, 2004.

[8] T. Hsiao, C. Lin, and H. Kenny Chiang, "Partial least squares learning regression for backpropagation network," In Proceedings of the 22d annual EMBS international conference, Chicago ,pp. 975-977, 2000.

[9] A.Debiolles, L.Oukhellou, and P.Aknin, "Combined use of partial least squares regression and neural network for diagnosis tasks, 'In Proceedings of the $17^{\text {th }}$ international conference on pattern recognition (ICPR'04), vol. 4, pp. 23-26, 2004.

[10] F. Leung, K. Lam, H. Ling, and S. Tam, "Tuning of the structure and parameters of a neural network using an improved genetic algorithm," IEEE Trans. Neural Netw., 14(1), pp. 79-88, 2003.

[11] J. Tsai, J. Chou, and T. Liu, "Tuning the structure and parameters of a neural network by using hybrid Taguchi-genetic algorithm," IEEE Trans. Neural Netw., 17(1), pp. 69-80, 2006.

[12] M.Fallahnezhad, M.Moradi, and S.Zaferanlouei, "A Hybrid Higher Order Neural Classifier for handling classification problems," Expert Systems with Applications, vol. 38, pp. 386-393, 2011.

[13] J. Lu, S. Chen, W. Wang, and H.Zuylen, "A hybrid model of partial least squares and neural network for traffic incident detection," Expert Systems with Applications ,vol. 39, pp. 4775-4784, 2012.

[14] L.Tzyy-Chyang, Y.Gwo-Ruey, and J. Jyh-Ching, "Quantum-Based Algorithm for Optimizing Artificial Neural Networks," IEEE transactions on neural networks and learning systems, 24(8), 2013.

[15] IAEA Technical Document, "Research Reactor Core Conversion Guidebook”. IAEA TECDOC-643, 1992.
[16] K. Ibrahim, H.Mazrou, T.Hamidouche, and H.Benkharfia, "MUDICO2D: A Two-Dimensional Multigroupe Diffusion Code for Perturbation Calculation in Light Water Research Reactors". In Proceedings of International Conference on the New Frontiers of Nuclear Technology: Reactor Physics, Safety and High-Performance Computing, PHYSOR, Seoul, Korea, 2002.

[17] H.Mazrou, "Performance improvement of artificial neural networks designed for safety key parameters prediction in nuclear research reactors". Nuclear Engineering and Design, vol. 239, pp. 1901-1910, 2009. 